TRANSACTIONS OF THE

AMERICAN MATHEMATICAL SOCIETY

Volume 350, Number 4, April 1998, Pages 1643-1661

S $0002-9947(98) 01928-X$

\title{
GEOMETRIC PROPERTIES OF THE DOUBLE-POINT DIVISOR
}

\author{
BO ILIC
}

\begin{abstract}
The locus of double points obtained by projecting a variety $X^{n} \subset$ $\mathbf{P}^{N}$ to a hypersurface in $\mathbf{P}^{n+1}$ moves in a linear system which is shown to be ample if and only if $X$ is not an isomorphic projection of a Roth variety. Such Roth varieties are shown to exist, and some of their geometric properties are determined.
\end{abstract}

\section{INTRODUCTION}

Consider a nonsingular, nondegenerate, projective variety $X^{n} \subset \mathbf{P}^{N}$ of degree $d$ and codimension $N-n \geq 2$. One way to study $X$ is to find a new variety $Y$ and a surjective morphism $f: X \rightarrow Y$ and then study $X$ via this information. In projective geometry, there are two typical ways of doing this.

Firstly, we can project $X$ from a generic linear subspace $\Lambda \subset \mathbf{P}^{N}$ of dimension $N-n-1$ to obtain a finite surjective morphism $f: X \rightarrow \mathbf{P}^{n}$. The locus on $X$ where $f$ is not locally an isomorphism is the ramification divisor, $\mathcal{B}_{X, \Lambda}$. If $H$ is a hyperplane section of $X$ and $K_{X}$ is a canonical divisor on $X$, then $\mathcal{B}_{X, \Lambda} \sim$ $K_{X}+(n+1) H$. Thus by varying the generic center of projection $\Lambda$ we obtain linearly equivalent divisors, and so it makes sense to consider the linear system $\left|\mathcal{B}_{X}\right|$ without reference to a center of projection. L. Ein proved in [Ein] that $\left|\mathcal{B}_{X}\right|$ is very ample by showing that $\left|K_{X}+n H\right|$ is base point free. P. Ionescu, A.J. Sommese, and A. Van de Ven improved this result by showing that $\left|K_{X}+(n-1) H\right|$ is base point free except in a few well understood cases [Io, Theorem 1.4].

Secondly, we can project $X$ from a generic linear subspace $\Lambda \subset \mathbf{P}^{N}$ of dimension $N-n-2$, obtaining a finite birational morphism $f: X \rightarrow Y \subset \mathbf{P}^{n+1}$, where $\mathrm{Y}$ is a hypersurface. The locus on $X$ where $f$ is not an isomorphism is the double-point divisor, $\mathcal{C}_{X, \Lambda}$. Since $\mathcal{C}_{X, \Lambda} \sim(d-n-2) H-K_{X}$, by varying the generic center of projection $\Lambda$ we obtain linearly equivalent divisors, and so it makes sense to consider the linear system $\left|\mathcal{C}_{X}\right|$ without reference to a center of projection. D. Mumford proved that $\left|\mathcal{C}_{X}\right|$ is base point free [BM, Technical Appendix to Section 3, Part 4]. Thus it seems natural to wonder to what extent a result parallel to Ein's holds in this case. The main result of this paper is a classification of those varieties $X$ such that $\left|\mathcal{C}_{X}\right|$ is ample.

To state the classification theorem we need:

Received by the editors July 20, 1996.

1991 Mathematics Subject Classification. Primary 14N05, 14C20, 14J40.

Key words and phrases. Double-point divisor, Roth variety, Castelnuovo variety, secant variety, conductor, projection. 
Definition 3.1. Let $X^{n} \subset \mathbf{P}^{N}$ be a nonsingular, nondegenerate projective variety of codimension $N-n \geq 2$. Suppose that there is an $n+1$ dimensional rational normal scroll $S=S_{0,0, a_{1}, \ldots, a_{n-1}}$ with all $a_{i} \geq 1, \sum_{i=1}^{n-1} a_{i}=N-n$ and singular locus $L$ ( $L$ is a line) such that $L \subset X \subset S$. Then $X$ is a Roth variety.

Then the main classification theorem is:

Theorem 4.2. Let $X^{n} \subset \mathbf{P}^{N}$ be a nonsingular, nondegenerate variety of codimension $N-n \geq 2$. Let $\left|\mathcal{C}_{X}\right|$ denote the linear system determined by the double-point divisor on $X$. Then the following are equivalent:

(1) $\left|\mathcal{C}_{X}\right|$ is ample.

(2) $\left|\mathcal{C}_{X}\right|$ separates points.

(3) $X$ is not an isomorphic projection of a Roth variety.

If, in addition, $X$ is linearly normal, then (3) can be replaced by:

$\left(3^{\prime}\right) X$ is not a Roth variety.

The proof relies on techniques of projective geometry. The main geometric lemma is a classification of varieties with certain highly degenerate relative secant varieties:

Theorem 4.3. Let $X^{n} \subset \mathbf{P}^{N}$ be a nonsingular, nondegenerate variety of codimension $N-n \geq 2$. Then the following are equivalent:

(1) There exists a positive dimensional subvariety $W$ of $X$ such that the secant variety $S(W, X)$ has dimension $n+1$.

(2) There is $p \neq q \in X$ such that $S(p, X)=S(q, X)$.

(3) $X$ is an isomorphic projection of a Roth variety.

If, in addition, $X$ is linearly normal, then (3) can be replaced by:

$\left(3^{\prime}\right) X$ is a Roth variety.

See the beginning of Section 2 for the definition of relative secant varieties.

We provide a convenient characterization and existence theorem for Roth varieties:

Theorem 3.8. Let $S^{n+1} \subset \mathbf{P}^{N}$ be a rational normal scroll $S_{0,0, a_{1}, \ldots, a_{n-1}}$ with all $a_{i} \geq 1$ and $\sum_{i=1}^{n-1} a_{i}=N-n$, and let $L$ be the singular locus of $S$. Let $\mathcal{E}=$ $\mathcal{O}_{\mathbf{P}^{1}} \oplus \mathcal{O}_{\mathbf{P}^{1}} \oplus \mathcal{O}_{\mathbf{P}^{1}}\left(a_{1}\right) \oplus \cdots \oplus \mathcal{O}_{\mathbf{P}^{1}}\left(a_{n-1}\right)$, and let $\pi_{1}: \mathbf{P}\left(\mathcal{E}^{*}\right) \rightarrow \mathbf{P}^{1}$ be a $\mathbf{P}^{n}$ bundle with diagram

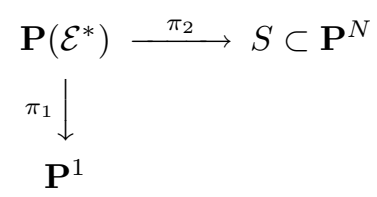

that desingularizes $S$ such that the morphism $\pi_{2}$ is given by the complete linear series $\left|\mathcal{O}_{\mathbf{P}\left(\mathcal{E}^{*}\right)}(1)\right|$. Let $H$ be the pullback of a hyperplane in $\mathbf{P}^{N}$ via $\pi_{2}$ and let $F$ be a fiber of $\pi_{1}$. Then for every $b>0$ there exists a nonsingular variety $\tilde{X} \in|b H+F|$ such that if $X=\pi_{2}(\tilde{X})$ then $\left.\pi_{2}\right|_{\tilde{X}}: \tilde{X} \rightarrow X$ is an isomorphism and $X$ is a linearly normal Roth variety of degree $b(N-n)+1$ (with associated scroll $S$ ). Conversely, if $X$ is a Roth variety of degree $d$ such that $L \subset X \subset S$, then $b=\frac{d-1}{N-n}$ is an integer and there is a desingularization of $S$ as above; $\pi_{2}: \mathbf{P}\left(\mathcal{E}^{*}\right) \rightarrow S$ and $\tilde{X} \in|b H+F|$, which is mapped isomorphically onto $X$ by $\pi_{2}$. (In particular, Roth varieties are linearly normal.) 
Historical background. Roth surfaces were studied by L. Roth in [Ro, §3.5], where he proved, for example, that such surfaces have maximal sectional genus. They were re-examined in modern language by A.J. Sommese in [So, §1.1] in order to settle "the main technical problem" in his first paper on the adjunction mapping. Sommese computed the sectional genus of a Roth surface and gave a geometric description of Roth surfaces as in Proposition 5.1 (2).

Restated in the language of projective geometry, he also proved

Lemma 1.1.1. Let $X \subset \mathbf{P}^{N}$ be a nonsingular, linearly normal surface. Let $L \subset X$ be a line and suppose that $H^{1}\left(X, \mathcal{O}_{X}\left(K_{X}+H-L\right)\right) \neq 0$. Then projection from the line $L$ extends to a morphism $\pi_{L}: X \rightarrow \mathbf{P}^{N-2}$ whose image is a rational normal curve of degree $N-2$.

From this theorem it is easy to deduce that $X$ is a Roth surface. This is the first theorem where the internal properties of $X$ were used to deduce the existence of a rational normal scroll $S_{0,0, N-2}$ containing $X$. Proposition 4.10 of this work follows the main line of argument of Sommese's proof of the above lemma.

Roth surfaces contained in $\mathbf{P}^{4}$ were extensively studied by K. Hulek, C. Okonek, and A. Van de Ven in [HOV]. Let $X \subset \mathbf{P}^{4}$ be a smooth surface. An ideal subsheaf $\mathcal{I}_{\tilde{X}} \subset \mathcal{I}_{X}$ determines a multiplicity-2 structure on $X$ if for each $p \in X$ there are local coordinates $x_{0}, \ldots, x_{3}$ such that $\left(I_{X}\right)_{p}=\left(x_{0}, x_{1}\right)$ and $\left(I_{\tilde{X}}\right)_{p}=\left(x_{0}, x_{1}^{2}\right)$. Their main theorem, somewhat restated, is

Theorem 13. Let $X^{2} \subset \mathbf{P}^{4}$ be a nonsingular Castelnuovo surface of degree $2 b+1$. Then $X$ is a Roth surface $\Longleftrightarrow$ there is a multiplicity-2 structure $\tilde{X}$ on $X$ such that $\tilde{X}$ is a complete intersection of type $(2,2 b+1)$.

They also proved that for every positive integer $b$ there exists a Roth surface of degree $2 b+1$ contained in $\mathbf{P}^{4}$ (Proposition 10). The proof of the general existence result in this paper (Theorem 3.8) is not a generalization of their proof.

This paper is a revised version of most of my Columbia University Ph.D. thesis [I]. I thank my advisor, Henry Pinkham, for his help and encouragement during my graduate studies. I also thank Rob Lazarsfeld for discussing an early version of this paper with me.

Conventions. 1. The ground field is the complex numbers.

2. If $V$ is a vector space then $\mathbf{P}(V)$ is the projective space of lines in $V$. The same convention extends to a vector bundle and its associated projective bundle.

3. If $X^{n} \subset \mathbf{P}^{N}$ is a variety and $p \in X$ is a nonsingular point of $X$, then $\tilde{T}_{p} X$ is the embedded projective tangent space to $X$ at $p$.

4. If $X$ is a subset of $\mathbf{P}^{N}$, then $\langle X\rangle$ is the smallest linear subspace of $\mathbf{P}^{N}$ that contains $X$. If $X$ and $Y$ are subsets of $\mathbf{P}^{N}$, then $\langle X, Y\rangle=\langle X \cup Y\rangle$.

5. The notation for rational normal scrolls and their basic projective geometric properties are taken from [Ha2]. Intersection theory on a rational normal scroll $S \subset \mathbf{P}^{N}$ and the description of $S$ as the image of a morphism $\tilde{S} \rightarrow S$ where $\tilde{S}$ is a projective bundle over $\mathbf{P}^{1}$ are discussed in [Ha1] and [EH]. For cohomological calculations on scrolls, see [Hart, Exercises III.8.1, III.8.4].

\section{Double-Point Divisors}

Definition and basic properties of relative secant varieties. Let $X^{n} \subset \mathbf{P}^{N}$ be a variety and let $W^{m} \subset X$ be a subvariety. Following F. Zak's terminology, we 
define the relative secant variety $S(W, X)$ as the special case of the join of $W$ and $X$ when $W \subset X$. Our definition is the specialization of the definition of join found in [Ha2, Example 8.1]. If $p \in X$, we write $S(p, X)$ for $S(\{p\}, X)$. If $p$ is a nonsingular point of $X$, then $S(p, X)=\left\{r \in \mathbf{P}^{N} \mid r\right.$ lies on a proper bisecant of $X$ through $p\} \cup \tilde{T}_{p} X$. In particular, $\tilde{T}_{p} X \subset S(p, X)$. Moreover, if $H$ is a hyperplane that meets $X$ transversally at a nonsingular point $p$, then $S(p, X \cap H)=S(p, X) \cap H$.

Basic properties of the double-point divisor. Let $X^{n} \subset \mathbf{P}^{N}$ be a nonsingular, nondegenerate variety of degree $d$ and codimension $N-n \geq 2$. Let $\Lambda$ be a linear subspace of $\mathbf{P}^{N}$ of dimension $N-n-2$ such that $X \cap \Lambda=\emptyset$. Let $\pi_{\Lambda}: X \rightarrow$ $\mathbf{P}^{n+1}$ denote the morphism obtained by restricting the projection with center $\Lambda$, $\mathbf{P}^{N}--\rightarrow \mathbf{P}^{n+1}$, to $X . \pi_{\Lambda}$ is a finite morphism and so $Y=\pi_{\Lambda}(X)$ is a hypersurface in $\mathbf{P}^{n+1}$. $\pi_{\Lambda}$ need not be birational onto its image, but for a generic choice of $\Lambda$ it is.

If $p \in X$ and $q=\pi_{\Lambda}(p)$, then we say that $\pi_{\Lambda}: X \rightarrow Y$ is an isomorphism at $p$ if there is an open neighborhood $U$ of $q$ such that

$$
\left.\pi_{\Lambda}\right|_{\pi_{\Lambda}^{-1}(U)}: \pi_{\Lambda}^{-1}(U) \rightarrow U
$$

is an isomorphism. $\pi_{\Lambda}$ is an isomorphism at $p$ if and only if $\langle\Lambda, p\rangle \cap X=\{p\}$ and $\tilde{T}_{p} X \cap \Lambda=\emptyset$. The first condition says that $\pi_{\Lambda}^{-1}(q)$ is a single point and the second one says that $\pi_{\Lambda}$ does not ramify at $p$. Equivalently, $\pi_{\Lambda}$ is an isomorphism at $p$ if and only if $S(p, X) \cap \Lambda=\emptyset$.

If $\pi_{\Lambda}$ is birational there is a divisor $\mathcal{C}_{X, \Lambda}$ on $X$, the double-point divisor, which depends on $X$ and $\Lambda$ such that:

(1) $\operatorname{support}\left(\mathcal{C}_{X, \Lambda}\right)=\left\{p \in X \mid \pi_{\Lambda}\right.$ is not an isomorphism at $\left.p\right\}=\{p \in X \mid$ $S(p, X) \cap \Lambda \neq \emptyset\}$.

(2) $\mathcal{C}_{X, \Lambda} \sim(d-n-2) H-K_{X}$.

(3) The sheaf of $\mathcal{O}_{Y}$ ideals $\left(\pi_{L}\right)_{*} \mathcal{O}_{X}\left(-\mathcal{C}_{X, \Lambda}\right)$ is the conductor of the finite birational map $\pi_{\Lambda}: X \rightarrow Y$.

For these basic properties of the double-point divisor see [BM, Technical Appendix to Section 3, Part 4] and [Kl, Sections I.D and V.A].

By (2) the divisor class of $\mathcal{C}_{X, \Lambda}$ does not depend on the center of projection $\Lambda$, so it makes sense to consider the linear system $\left|\mathcal{C}_{X}\right|$ without reference to a given $\Lambda$.

Remark 2.1. If $X^{n} \subset \mathbf{P}^{N}$ is a variety, $\Lambda^{m}$ is a center of projection such that $\Lambda \cap X=\emptyset$ and $\pi_{\Lambda}: X \rightarrow \mathbf{P}^{N-m-1}$ is the projection, then let $Y=\pi_{\Lambda}(X)$. If $\pi_{\Lambda}: X \rightarrow Y$ is an isomorphism, we say that $Y$ is an isomorphic projection of $X$. If this is so, then $\mathcal{O}_{X}\left(\mathcal{C}_{X}\right) \cong \mathcal{O}_{Y}\left(\mathcal{C}_{Y}\right)$. Thus, for example, $X^{n} \subset \mathbf{P}^{N}$ is a nonsingular, nondegenerate variety such that $\left|\mathcal{C}_{X}\right|$ separates points $\Longleftrightarrow X$ is an isomorphic projection of a linearly normal variety $\tilde{X}$ such that $\left|\mathcal{C}_{\tilde{X}}\right|$ separates points.

Lemma 2.2. If $p, q \in X$ and $S(p, X) \neq S(q, X)$, then there exists an $N-n-2$ plane $\Lambda$ such that $\Lambda \cap S(p, X)=\emptyset$ but $\Lambda \cap S(q, X) \neq \emptyset . \quad\left(S o \mathcal{C}_{X, \Lambda}\right.$ is defined and $p \notin \mathcal{C}_{X, \Lambda}$ but $\left.q \in \mathcal{C}_{X, \Lambda}\right)$.

Proof. Let $M$ be an $N-n-1$ plane that intersects both $S(q, X)$ and $S(p, X)$ in a finite collection of points (the generic $N-n-1$ plane does this) and such that there is a point $r \in(M \cap S(q, X)) \backslash S(p, X)$. If we cannot do this, then $S(q, X) \subset S(p, X)$, which is a contradiction. Let $\Lambda$ be the $N-n-2$ plane determined by a hyperplane in $M$ which contains $r$ but avoids the finite set of points $M \cap S(p, X) . \Lambda$ satisfies the requirements of the lemma. 
Corollary 2.3. If $\left|\mathcal{C}_{X}\right|$ does not separate the points $p$ and $q$, then $S(p, X)=$ $S(q, X)$.

The next corollary follows by the Kleiman-Nakai-Moishezon criterion for ampleness.

Corollary 2.4. If $\left|\mathcal{C}_{X}\right|$ separates points then $\left|\mathcal{C}_{X}\right|$ is ample.

Proposition 2.5. If $W$ is a positive dimensional subvariety of $X$ such that $S(p, X)$ $=S(W, X)$ for all $p \in W$, then $S(W, X)=\bigcup_{p \in W} \tilde{T}_{p} X$.

Proof. $\tilde{T}_{p} X \subset S(W, X)$ for all $p \in W$. So $T=\bigcup_{p \in W} \tilde{T}_{p} X$ is subvariety of $S(W, X)$. Since $S(W, X)$ is $n+1$ dimensional and irreducible, if $T \neq S(W, X)$ then $\tilde{T}_{p} X=\tilde{T}_{q} X$ for all $p, q \in W$. Since $\operatorname{dim}(W) \geq 1$, this contradicts Zak's theorem on tangencies [Zak, Chapter 1, Corollary 1.8].

Proposition 2.6. If $W$ is a possibly reducible subvariety of $X$ such that $S(p, X)=$ $S(W, X)$ for all $p \in W$, then $W \subset \bigcap_{p \in W} \tilde{T}_{p} X$. Moreover, if $W$ is positive dimensional, then $\operatorname{dim}\left(\bigcap_{p \in W} \tilde{T}_{p} X\right) \leq n-1$.

Proof. Pick $p \in W$. We'll show that $W \subset \tilde{T}_{p} X$. Let $q \in W$. Suppose that $q \notin \tilde{T}_{p} X$. Then we can "define" a map:

$$
\tilde{T}_{p} X \backslash\left(\tilde{T}_{q} X \cup X\right) \rightarrow\left\langle\tilde{T}_{p} X, q\right\rangle
$$

where a point $r$ goes to a point $w \in X, w \neq q$, on the line $\langle q, r\rangle$. Observe that:

(1) For each $r \in \tilde{T}_{p} X, r \in S(p, X)$ and thus $r \in S(q, X)$; and since $r \notin \tilde{T}_{q} X$, $\langle q, r\rangle$ is a proper bisecant of $X$.

(2) The map is not well defined since there could be more than one choice of $w$, but complex analytic locally, the map can be defined to be analytic; use the implicit function theorem on the natural incidence relation.

(3) The map is injective.

Thus we have an $n$ dimensional complex neighborhood of $X \subset\left\langle\tilde{T}_{p} X, q\right\rangle$. Thus, $X \subset\left\langle\tilde{T}_{p} X, q\right\rangle$, which contradicts the nondegeneracy of $X$. Thus, $q \in \tilde{T}_{p} X$. Since $q \in W$ was arbitrary, $W \subset \tilde{T}_{p} X$. Since $p \in W$ was arbitrary, the first part of the proposition follows.

If $\operatorname{dim}\left(\bigcap_{p \in W} \tilde{T}_{p} X\right)=n$, then $\tilde{T}_{p} X=\tilde{T}_{q} X$ for all $p, q \in W$. But since $\operatorname{dim}(W) \geq$ 1 , this contradicts Zak's theorem on tangencies.

Proposition 2.7. Let $X \subset \mathbf{P}^{N}$ be a nondegenerate, nonsingular curve of codimension $N-1 \geq 2$. Then $\left|\mathcal{C}_{X}\right|$ is very ample.

Proof. By taking a generic projection, we can assume without loss of generality that $X \subset \mathbf{P}^{3}$ (see Remark 2.1). First we'll show that $\left|\mathcal{C}_{X}\right|$ separates points. Pick $p, q \in X, p \neq q$. Pick $r \in X$ not on the line $\langle p, q\rangle$ and consider the line $L=\langle q, r\rangle$. If there exists $w \in L \backslash X$ such that $w \notin S(p, X)$, then $\mathcal{C}_{X, w}$ is defined and $p \notin \mathcal{C}_{X, w}$, but $q \in \mathcal{C}_{X, w}$. Thus, suppose that $w \in S(p, X)$ for all $w \in L \backslash X$. Then for generic $w \in L,\langle w, p\rangle$ is a proper bisecant of $X$. Thus varying $w$ we see that the plane $\langle L, p\rangle$ contains infinitely many points of $X$, so that $X \subset\langle L, p\rangle$. But this contradicts the nondegeneracy of $X$.

Now we'll show that $\left|\mathcal{C}_{X}\right|$ separates tangent directions, i.e., given $q \in X$, there exists $w \in \mathbf{P}^{N}$ such that $\left|\mathcal{C}_{X, w}\right|$ is defined, $q \in \mathcal{C}_{X, w}$, but $w \notin \tilde{T}_{q} X$. Clearly we can 
pick $r \in X$ such that $\langle q, r\rangle$ is a bisecant of $X$ and $r \notin \tilde{T}_{q} X$. Then there must be some $w \in\langle q, r\rangle$ that works, or we get a contradiction as above.

The last proposition can also be proven by using Castelnuovo's bound for the genus of a space curve [Hart, Theorem IV.6.4]. If $g$ is the genus of $X$, then one shows that $\operatorname{deg}\left(\mathcal{C}_{X}\right)=\operatorname{deg}\left((d-3) H-K_{X}\right) \geq 2 g+1$, and so $\mathcal{C}_{X}$ is very ample.

Recall that a variety $X^{n} \subset \mathbf{P}^{N}$ is semi-canonical if $K_{X} \sim \alpha H$ for some integer $\alpha$. For example, by the Barth-Larsen theorem [Lar], if $n \geq(N-n)+2$ then $X$ is semi-canonical. Also, by using the adjunction formula, we see that complete intersections are semi-canonical.

Proposition 2.8. Let $X^{n} \subset \mathbf{P}^{N}$ be a nonsingular, nondegenerate, semi-canonical variety of codimension $N-n \geq 2$. Then $\left|\mathcal{C}_{X}\right|$ is very ample.

Proof. Let $d$ be the degree of $X$. Then $\mathcal{C}_{X} \sim(d-n-2) H-K_{X} \sim \alpha H$ for some integer $\alpha$. We can choose an $N-n-2$ plane $\Lambda$ in $\mathbf{P}^{N}$ such that $\mathcal{C}_{X, \Lambda}$ is an element of $\left|\mathcal{C}_{X}\right|$. Hence $H^{0}\left(X, \mathcal{O}_{X}\left(\mathcal{C}_{X}\right)\right) \neq 0$, and so $\alpha \geq 1$. Thus $\left|\mathcal{C}_{X}\right|$ is very ample.

\section{Roth VARIETIES}

Definition 3.1. Let $X^{n} \subset \mathbf{P}^{N}$ be a nonsingular, nondegenerate projective variety of codimension $N-n \geq 2$. Suppose that there is an $n+1$ dimensional rational normal scroll $S=S_{0,0, a_{1}, \ldots, a_{n-1}}$ with all $a_{i} \geq 1, \sum_{i=1}^{n-1} a_{i}=N-n$ and singular locus $L$ ( $L$ is a line) such that $L \subset X \subset S$. Then $X$ is a Roth variety.

Let $S$ be the rational normal scroll $S_{0,0, a_{1}, \ldots, a_{n-1}} \subset \mathbf{P}^{N}=\mathbf{P}(V)$, where $V$ is an $N+1$ dimensional vector space and all $a_{i} \geq 1$. Then $\operatorname{dim}(S)=n+1$, $d_{S}:=\operatorname{deg}(S)=\sum_{i=1}^{n-1} a_{i}$, and $N=d_{S}+n$. Let $L$ be the singular locus of $S$. $L$ is a line contained in $S$. Then $S$ can be considered as the cone with vertex $L$ over the nonsingular rational normal scroll $S_{a_{1}, a_{2}, \ldots, a_{n-1}}$, where $\left\langle S_{a_{1}, a_{2}, \ldots, a_{n-1}}\right\rangle$ and $L$ are complementary linear subspaces of $\mathbf{P}^{N}$.

$S$ can also be described geometrically as follows: there are linear subspaces $V_{0}, V_{1}, \ldots, V_{n-1}$ of $V$ such that:

(1) $V_{i} \cap V_{j}=0$ for all $i$ and $j$,

(2) $V=V_{0} \oplus V_{1} \oplus \cdots \oplus V_{n-1}$,

(3) $\operatorname{dim}\left(V_{0}\right)=2$,

(4) $\operatorname{dim}\left(V_{i}\right)=a_{i}+1$ if $i \geq 1$,

and such that for each $i \geq 1$ we can find a rational normal curve $C_{i}$ of degree $a_{i}$ in $\mathbf{P}\left(V_{i}\right)$ and isomorphisms $\phi_{i}: \mathbf{P}^{1} \rightarrow C_{i}$ such that:

$$
S=\bigcup_{p \in \mathbf{P}^{1}}\left\langle L, \phi_{1}(p), \phi_{2}(p), \ldots, \phi_{n-1}(p)\right\rangle .
$$

Note also that if $p \neq q$, then

$$
\left\langle L, \phi_{1}(p), \phi_{2}(p), \ldots, \phi_{n-1}(p)\right\rangle \cap\left\langle L, \phi_{1}(q), \phi_{2}(q), \ldots, \phi_{n-1}(q)\right\rangle=L .
$$

Let $\tilde{S} \subset \mathbf{P}^{1} \times \mathbf{P}^{N}$ be defined by

$$
\tilde{S}=\left\{\left(p,\left\langle L, \phi_{1}(p), \phi_{2}(p), \ldots \phi_{n-1}(p)\right\rangle\right) \mid p \in \mathbf{P}^{1}\right\} .
$$


$\tilde{S}$ is a variety, and if

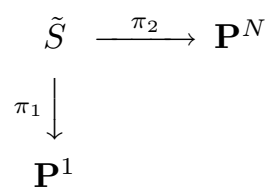

are the natural projections, then $\pi_{1}: \tilde{S} \rightarrow \mathbf{P}^{1}$ makes $\tilde{S}$ into a $\mathbf{P}^{n}$-bundle over $\mathbf{P}^{1}$ and hence $\tilde{S}$ is nonsingular. Note also that $\pi_{2}(\tilde{S})=S$. Moreover, $\pi_{2}: \tilde{S} \rightarrow S$ is such that $\tilde{S} \backslash \pi_{2}^{-1}(L) \rightarrow S \backslash L$ is an isomorphism. Thus $\pi_{2}$ is birational onto its image. Finally, $\pi_{2}^{-1}(L)=\mathbf{P}^{1} \times L \subset \tilde{S}$.

Let $F$ denote a fiber of $\pi_{1}$ and $H$ denote the pullback of a hyperplane section given by the map $\pi_{2}: \tilde{S} \rightarrow \mathbf{P}^{N}$. In fact, if $\mathcal{E}=\mathcal{O}_{\mathbf{P}^{1}} \oplus \mathcal{O}_{\mathbf{P}^{1}} \oplus \mathcal{O}_{\mathbf{P}^{1}}\left(a_{1}\right) \oplus \cdots \oplus$ $\mathcal{O}_{\mathbf{P}^{1}}\left(a_{n-1}\right)$, then $\tilde{S}=\mathbf{P}\left(\mathcal{E}^{*}\right), \pi_{1}$ is the natural projection to $\mathbf{P}^{1}$, and $\mathcal{O}_{\mathbf{P}\left(\mathcal{E}^{*}\right)}(1)=$ $\mathcal{O}_{\tilde{S}}(H)$. Let $A(\tilde{S})$ be the ring of cycles mod rationally equivalent cycles (the Chow ring). Then

$$
A(\tilde{S})=\mathbf{Z}[F, H] /\left(F^{2}, H^{n+1}-d_{S} H^{n} \cdot F, H^{n+2}, H^{n+1} \cdot F\right) .
$$

Also $\operatorname{deg}\left(H^{n} \cdot F\right)=1$ and $\operatorname{deg}\left(H^{n+1}\right)=d_{S}$. Recall also that

$$
K_{\tilde{S}} \sim-(n+1) H+\left(d_{S}-2\right) F .
$$

$[\mathrm{EH}]$ is a reference for these facts.

Lemma 3.2. In $A(\tilde{S}), \mathbf{P}^{1} \times L \sim H^{n-1}-d_{S} H^{n-2} \cdot F$.

Proof. We know that $\mathbf{P}^{1} \times L \sim \alpha H^{n-1}+\beta H^{n-2} \cdot F$ for some integers $\alpha$ and $\beta$. Now by the construction we see that $\left(\mathbf{P}^{1} \times L\right) \cdot F \cdot H=1$. Thus $1=\alpha H^{n} \cdot F+\beta H^{n-1} \cdot F^{2}=$ $\alpha H^{n} \cdot F=\alpha$, so that $\alpha=1$. Also, $\left(\mathbf{P}^{1} \times L\right) \cdot H^{2}=0$ since $\pi_{2}\left(\mathbf{P}^{1} \times L\right)=L$. Thus, $0=H^{n+1}+\beta H^{n} \cdot F$, and so $\beta=-d_{S}$.

We have the diagram of projections obtained by restriction to $\mathbf{P}^{1} \times L$

$$
\begin{gathered}
\mathbf{P}^{1} \times L \stackrel{\left.\pi_{2}\right|_{\mathbf{P}^{1} \times L}}{\longrightarrow} L \\
\left.\pi_{1}\right|_{\mathbf{P}^{1} \times L} \downarrow \\
\mathbf{P}^{1}
\end{gathered}
$$

The fibers over $\mathbf{P}^{1}$ are linearly equivalent in $A\left(\mathbf{P}^{1} \times L\right)$ and thus are rationally equivalent in $A(\tilde{S})$. The same holds for fibers over $L$. Let $B$ be a fiber over $\mathbf{P}^{1}$ and let $C$ be a fiber over $L$.

Lemma 3.3. In $A(\tilde{S}), B \sim H^{n-1} \cdot F$ and $C \sim H^{n}-d_{S} H^{n-1} \cdot F$.

Proof. $B \sim\left(\mathbf{P}^{1} \times L\right) \cdot F=\left(H^{n-1}-d_{S} H^{n-2} \cdot F\right) \cdot F=H^{n-1} \cdot F$ and $C \sim\left(\mathbf{P}^{1} \times L\right) \cdot H=$ $\left(H^{n-1}-d_{S} H^{n-2} \cdot F\right) \cdot H$.

Let $b$ be a positive integer. Consider the linear system $|b H+F|$ on $\tilde{S}$. $|H|$ is clearly base point free. $|H+F|$ is very ample, since $H+F$ is the pullback of a hyperplane section of the projective embedding of $\tilde{S}$ as the rational normal scroll $S_{1,1, a_{1}+1, \ldots, a_{n-1}+1}$. Thus $|b H+F|$ is very ample. Hence, by Bertini's theorem, a generic $\tilde{X} \in|b H+F|$ is a smooth and irreducible $n$ dimensional variety and intersects $\mathbf{P}^{1} \times L$ in an irreducible curve.

Now choose an $\tilde{X}$ as above and let $X=\pi_{2}(\tilde{X})$. 
Proposition 3.4. The restriction of $\pi_{2}$ to $\tilde{X}$ gives an isomorphism $\tilde{X} \cong X$.

Proof. The restriction of $\pi_{2}$ gives an isomorphism $\tilde{X} \backslash\left(\mathbf{P}^{1} \times L\right) \rightarrow X \backslash L$. I claim that $\left.\pi_{2}\right|_{\tilde{X}}: \tilde{X} \rightarrow X$ is a bijection: If not, there is a point $p \in L$ such that $\left(\mathbf{P}^{1} \times p\right) \cap \tilde{X}$ contains more than one point. But,

$$
\tilde{X} \cdot C=(b H+F) \cdot\left(H^{n}-d_{S} H^{n-1} \cdot F\right)=b H^{n+1}-b d_{S} H^{n} \cdot F+H^{n} \cdot F=1 .
$$

So either $\mathbf{P}^{1} \times p$ intersects $\tilde{X}$ in exactly one point $q$ and the intersection is transversal at $q$, or $\mathbf{P}^{1} \times p$ is entirely contained within $\tilde{X}$. Suppose the second case occurs. Then $\left(\mathbf{P}^{1} \times L\right) \cdot \tilde{X}=\mathbf{P}^{1} \times p \sim C$, since we know that $\mathbf{P}^{1} \times L$ meets $\tilde{X}$ in an irreducible curve. Now,

$$
\begin{aligned}
\left(\mathbf{P}^{1} \times L\right) \cdot \tilde{X} \cdot H & =\left(H^{n-1}-d_{S} H^{n-2} \cdot F\right) \cdot(b H+F) \cdot H \\
& =b H^{n+1}+\left(1-b d_{S}\right) H^{n} \cdot F=1 .
\end{aligned}
$$

On the other hand, $C \cdot H=0$. This contradiction shows that the second case doesn't occur, and so our map is a bijection.

Take any point $p \in L$ and let $q$ be the unique point of intersection of $\mathbf{P}^{1} \times p$ with $\tilde{X}$. To show that our map is an isomorphism it suffices to show that $\left.\pi_{2}\right|_{\tilde{X}}$ is immersive at $q$, i.e. that $\left.d \pi_{2}\right|_{\tilde{X}}$ is injective at $q$ : In fact, the kernel of $\left.\left(d \pi_{2}\right)\right|_{q}: T_{q} \tilde{S} \rightarrow$ $T_{\pi_{2}(q)} \mathbf{P}^{N}$ is exactly the one-dimensional subspace $T_{q}\left(\mathbf{P}^{1} \times p\right)$ : clearly $T_{q}\left(\mathbf{P}^{1} \times p\right)$ is contained in the kernel since $\pi_{2}$ contracts $\mathbf{P}^{1} \times p$. On the other hand, $\pi_{2}$ acts as the identity map on $\mathbf{P}^{n}=\pi_{1}^{-1}\left(\pi_{1}(q)\right)$. Thus, since $\mathbf{P}^{1} \times p$ meets $\tilde{X}$ transversally at $q$, our claim follows.

Proposition 3.5. $X$ is a nonsingular, nondegenerate, and linearly normal variety.

Proof. $X$ is nonsingular, since it is isomorphic to $\tilde{X}$ and $\tilde{X}$ is nonsingular. A hyperplane containing $X$ pulls back to give a nonzero global section of $\mathcal{I}_{\tilde{X} / \tilde{S}}(H)$, where $\mathcal{I}_{\tilde{X} / \tilde{S}}$ denotes the sheaf of ideals of $\tilde{X}$ in $\tilde{S}$. Thus, to prove that $X$ is nondegenerate it suffices to show that $H^{0}\left(\tilde{S}, \mathcal{I}_{\tilde{X} / \tilde{S}}(H)\right)=0$. Assuming that $X$ is nondegenerate, to show that $X$ is linearly normal it suffices to show that the morphism $\tilde{X} \rightarrow X \subset \mathbf{P}^{N}=\mathbf{P}\left(H^{0}\left(\tilde{S}, \mathcal{O}_{\tilde{S}}(H)\right)\right)$ is given by a complete linear series. This follows if $H^{0}\left(\tilde{X}, \mathcal{O}_{\tilde{X}}(H)\right)=H^{0}\left(\tilde{S}, \mathcal{O}_{\tilde{S}}(H)\right)$, which follows if $H^{1}\left(\tilde{S}, \mathcal{I}_{\tilde{X} / \tilde{S}}(H)\right)=0$. Since $\mathcal{I}_{\tilde{X} / \tilde{S}}=\mathcal{O}_{\tilde{S}}(-b H-F)$, it suffices to show that $H^{i}\left(\tilde{S}, \mathcal{O}_{\tilde{S}}((1-b) H-F)\right)=0$ for $i=0,1$ and $b \geq 1$.

This divides into two cases: $b \geq 2$ and $b=1$. So suppose that $b \geq 2$. By Serre duality it suffices to show that $H^{i}\left(\tilde{S}, \mathcal{O}_{\tilde{S}}\left(K_{\tilde{S}}+((b-1) H+F)\right)\right)=0$ for $i=n, n+1$. Since $b \geq 2,|(b-1) H+F|$ is very ample and so the required vanishing follows from Kodaira's vanishing theorem.

If $b=1$ the above argument doesn't apply, but a direct cohomology computation for scrolls does the trick. I want to show that $H^{i}\left(\tilde{S}, \mathcal{O}_{\tilde{S}}(-F)\right)=0$ for $i=0,1$. But $\pi_{1 *} \mathcal{O}_{\tilde{S}}(-F)=\mathcal{O}_{\mathbf{P}^{1}}(-1)$ and $R^{i} \pi_{1 *} \mathcal{O}_{\tilde{S}}(-F)=0$ for $i>0$, and so $H^{i}\left(\tilde{S}, \mathcal{O}_{\tilde{S}}(-F)\right)=$ $H^{i}\left(\mathbf{P}^{1}, \mathcal{O}_{\mathbf{P}^{1}}(-1)\right)=0$ for $i=0,1$, as required.

Let $X^{n} \subset \mathbf{P}^{N}=\mathbf{P}(V)$ be a nonsingular, nondegenerate projective variety. Let $L^{m}=\mathbf{P}\left(V_{0}\right)$ be a linear space contained in $X$ and let $\pi_{L}: X--\rightarrow \mathbf{P}\left(V / V_{0}\right)$ be the rational map given by projection from the center $L$. Let $\Gamma \subset X \times \mathbf{P}\left(V / V_{0}\right)$ be 
the graph of $\pi_{L}$. We obtain the diagram

$$
\begin{array}{cccccc}
E & \subset & \Gamma & \stackrel{f_{2}}{\longrightarrow} & \mathbf{P}\left(V / V_{0}\right) \\
\downarrow & & f_{1} \downarrow & & \| \\
L & \subset & X & --\rightarrow & \mathbf{P}\left(V / V_{0}\right)
\end{array}
$$

where $\Gamma$ is the blowup of $X$ along the center $L \subset X$ and $E$ is the exceptional divisor of the blowup.

Thus, $\left.f_{1}\right|_{\Gamma \backslash E}: \Gamma \backslash E \rightarrow X \backslash L$ is an isomorphism, $f_{1}^{-1}(L)=E$, and the projection $\left.f_{1}\right|_{E}: E \rightarrow L$ makes $E$ into a $\mathbf{P}^{n-m-1}$ bundle over $L$. In particular, for each $p \in L$, $f_{1}^{-1}(p)$ is naturally identified with $\mathbf{P}\left(N_{p}(L / X)\right)$, where $N_{p}(L / X)=T_{p} X / T_{p} L$ is the normal space of $L$ in $X$ at $p$. With this identification $\left.f_{2}\right|_{f_{1}^{-1}(p)}$ is just the linear inclusion $\mathbf{P}\left(N_{p}(L / X)\right) \hookrightarrow \mathbf{P}\left(V / V_{0}\right)$.

Pick a linear subspace $V_{1}$ of $V$ complementary to $V$, i.e., so that $V=V_{0} \oplus V_{1}$ and $\operatorname{dim}\left(V_{1}\right)=N-m$. Then the projection can be described as follows: if $p \in X \backslash L=$ $\Gamma \backslash E$, then $\pi_{L}(p)$ is the unique point of intersection of $\langle L, p\rangle$ with $\mathbf{P}\left(V_{1}\right)$. If $p \in E$, then let $q=f_{1}(p)$ and let $v \in \mathbf{P}\left(N_{q}(L / X)\right) \subset \mathbf{P}\left(V_{1}\right)$ be the normal direction at $q$ determined by $p$. Then $\langle L, v\rangle$ is a well defined $m+1$ dimensional linear subspace of $\mathbf{P}(V)$ and $f_{2}(p)$ is the unique point of intersection of $\langle L, v\rangle$ with $\mathbf{P}\left(V_{1}\right)$.

Now let $X^{n} \subset \mathbf{P}^{N}=\mathbf{P}(V)$ be a Roth variety with $S=S_{0,0, a_{1}, \ldots, a_{n-1}}$ the rational normal scroll having singular locus $L$ such that $L \subset X \subset S$. Desingularize the scroll $S$ as in the above construction to obtain the diagram

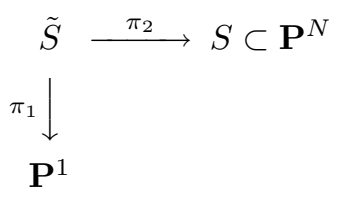

Let $\tilde{X}=\overline{\pi_{2}^{-1}(X \backslash L)}$.

Claim 3.6. If $S \neq S_{0,0,1,1}$, then $\left.\pi_{2}\right|_{\tilde{X}}: \tilde{X} \rightarrow X$ is an isomorphism. If $S=S_{0,0,1,1}$, there are two possible ways to desingularize $S$ by the above construction, corresponding to the two rulings of $S_{1,1} \cong \mathbf{P}^{1} \times \mathbf{P}^{1}$. For one of them $\tilde{X}$ is isomorphic to $X$, and for the other $\tilde{X}$ is the blow up of $X$ along $L$.

Proof of Claim. Identify $\mathbf{P}\left(V / V_{0}\right)$ with $\mathbf{P}^{N-2}=\mathbf{P}\left(V_{1} \oplus \cdots \oplus V_{n-1}\right) \subset \mathbf{P}(V)=\mathbf{P}^{N}$. Now let $W \subset \mathbf{P}^{1} \times \mathbf{P}^{N-2}$ be defined by

$$
W=\left\{\left(p,\left\langle\phi_{1}(p), \ldots, \phi_{n-1}(p)\right\rangle\right) \mid p \in \mathbf{P}^{1}\right\}
$$

and consider the diagram of natural projections

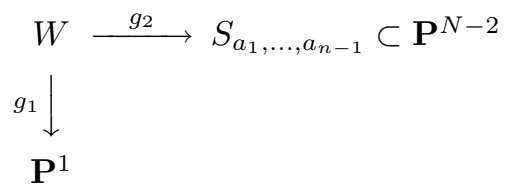

$g_{2}$ is an isomorphism and $g_{1}$ makes $W$ into a $\mathbf{P}^{n-2}$-bundle over $\mathbf{P}^{1}$.

Let $\pi_{L}: X--\rightarrow \mathbf{P}^{N-2}$ be the rational map given by projection from $L$. Since $X \subset S, \pi_{L}(X) \subset S_{a_{1}, \ldots, a_{n-1}}$; thus if $\Gamma$ is the graph of $\pi_{L}$, then $\Gamma \subset X \times S_{a_{1}, \ldots, a_{n-1}}$ 
with diagram of projections

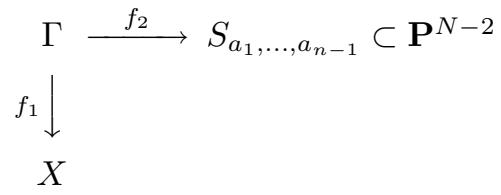

We obtain a morphism $h: \Gamma \rightarrow \mathbf{P}^{1} \times \mathbf{P}^{N}$ via the composition

$$
\Gamma \subset X \times S_{a_{1}, \ldots, a_{n-1}} \stackrel{\left(i d, g_{1} \circ g_{2}^{-1}\right)}{\longrightarrow} X \times \mathbf{P}^{1} \stackrel{\text { interchange }}{\longrightarrow} \mathbf{P}^{1} \times X \subset \mathbf{P}^{1} \times \mathbf{P}^{N}
$$

such that $h(\Gamma)=\tilde{X}$ and the diagram

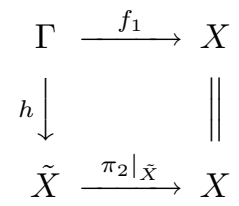

is commutative.

Now $\Gamma \backslash E \cong X \backslash L \cong \tilde{X} \backslash \mathbf{P}^{1} \times L$. If $p \in L$ then $f_{1}^{-1}(p)=p \times \mathbf{P}\left(N_{p}(L / X)\right)$. Then $h\left(f_{1}^{-1}(p)\right)$ is a point $\Longleftrightarrow g_{1} \circ g_{2}^{-1}\left(\mathbf{P}\left(N_{p}(L / X)\right)\right)$ is a point $\Longleftrightarrow g_{2}^{-1}\left(\mathbf{P}\left(N_{p}(L / X)\right)\right)$ is a fiber of the $\mathbf{P}^{n-2}$ bundle $g_{1}: W \rightarrow \mathbf{P}^{1}$. But any $n-2$ dimensional linear space on a nonsingular $n-1$ dimensional scroll must be a fiber as above unless the scroll is $S_{1,1}$, in which case it could also be a fiber for the other possible way of making $S_{1,1}$ into a $\mathbf{P}^{1}$ bundle via the above construction. Thus if $S \neq S_{0,0,1,1}$, then $\left.\pi_{2}\right|_{\tilde{X}}: \tilde{X} \rightarrow X$ is a birational bijective morphism and is thus an isomorphism by Zariski's main theorem [Mum, Section III.9, Proposition 1]. If $S=S_{0,0,1,1}$, then by Zariski's main theorem either $\left.\pi_{2}\right|_{\tilde{X}} ^{-1}(L)=\mathbf{P}^{1} \times L$ or $\left.\pi_{2}\right|_{\tilde{X}}: \tilde{X} \rightarrow X$ is bijective and thus an isomorphism. In the first case, $h: \Gamma \rightarrow \tilde{X}$ is a bijective morphism. It is easy to see that $h$ is an immersion, and so it is an isomorphism. Thus $\tilde{X}$ is the blow up of $X$ along $L$. In either case we see that $\mathbf{P}\left(N_{p}(L / X)\right) \cdot \mathbf{P}\left(N_{q}(L / X)\right)=0$ in $A\left(S_{1,1}\right)$ for $p, q \in L$, and thus, switching to the other desingularization for $S$, we switch between the two possibilities for $\tilde{X}$.

By the claim we can assume that $\left.\pi_{2}\right|_{\tilde{X}}: \tilde{X} \rightarrow X$ is an isomorphism. In particular, $\tilde{X} \cdot C=1$. Now $\tilde{X}=\alpha H+\beta F$ for some integers $\alpha$ and $\beta$. Then

$$
\begin{aligned}
\tilde{X} \cdot C & =(\alpha H+\beta F) \cdot\left(H^{n}-d_{S} H^{n-1} \cdot F\right) \\
& =\alpha H^{n+1}+\left(\beta-\alpha d_{S}\right) H^{n} \cdot F=\beta,
\end{aligned}
$$

and so $\beta=1$. Finally, $d=\operatorname{deg}(X)=H^{n} \cdot \tilde{X}=\alpha d_{S}+1$, and so $\alpha=\frac{d-1}{N-n}$. To sum up, we have proved

Theorem 3.7. Let $S^{n+1} \subset \mathbf{P}^{N}$ be a rational normal scroll $S_{0,0, a_{1}, \ldots, a_{n-1}}$ with all $a_{i} \geq 1$ and $\sum_{i=1}^{n-1} a_{i}=N-n$, and let $L$ be the singular locus of $S$. Let $\mathcal{E}=$ $\mathcal{O}_{\mathbf{P}^{1}} \oplus \mathcal{O}_{\mathbf{P}^{1}} \oplus \mathcal{O}_{\mathbf{P}^{1}}\left(a_{1}\right) \oplus \cdots \oplus \mathcal{O}_{\mathbf{P}^{1}}\left(a_{n-1}\right)$, and let $\pi_{1}: \mathbf{P}\left(\mathcal{E}^{*}\right) \rightarrow \mathbf{P}^{1}$ be a $\mathbf{P}^{n}$ bundle with diagram

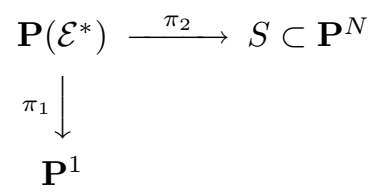


that desingularizes $S$ such that the morphism $\pi_{2}$ is given by the complete linear series $\left|\mathcal{O}_{\mathbf{P}\left(\mathcal{E}^{*}\right)}(1)\right|$. Let $H$ be the pullback of a hyperplane in $\mathbf{P}^{N}$ via $\pi_{2}$ and let $F$ be a fiber of $\pi_{1}$. Then for every $b>0$ there exists a nonsingular variety $\tilde{X} \in|b H+F|$ such that if $X=\pi_{2}(\tilde{X})$ then $\left.\pi_{2}\right|_{\tilde{X}}: \tilde{X} \rightarrow X$ is an isomorphism and $X$ is a linearly normal Roth variety of degree $b(N-n)+1$ (with associated scroll $S$ ). Conversely, if $X$ is a Roth variety of degree $d$ such that $L \subset X \subset S$, then $b=\frac{d-1}{N-n}$ is an integer and there is a desingularization of $S$ as above; $\pi_{2}: \mathbf{P}\left(\mathcal{E}^{*}\right) \rightarrow S$ and $\tilde{X} \in|b H+F|$ which is mapped isomorphically onto $X$ by $\pi_{2}$. (In particular, Roth varieties are linearly normal.)

Note that further properties of Roth varieties are given in Proposition 3.14.

Now let $d=\operatorname{deg}(X)$. Then $d=H^{n} \cdot \tilde{X}=H^{n} \cdot(b H+F)=b d_{S}+1$. Let $\mathcal{C}_{X}$ denote a double-point divisor of $X \subset \mathbf{P}^{N}$.

Proposition 3.8. $\mathcal{C}_{X} \cdot L=0$. Thus $\left|\mathcal{C}_{X}\right|$ is not ample.

Proof. $\mathcal{C}_{X} \cdot L=\left((d-n-2) H-K_{X}\right) \cdot L$. Since $\tilde{X}$ is isomorphic to $X$, we can write the intersection occurring in $A(X)$ as an intersection occurring in $A(\tilde{X})$. Thus,

$$
\mathcal{C}_{X} \cdot L=\left((d-n-2) H-K_{\tilde{X}}\right) \cdot\left(\left.\pi_{2}\right|_{\tilde{X}}\right)^{-1}(L) .
$$

$\left(\left.\pi_{2}\right|_{\tilde{X}}\right)^{-1}(L)$ is rationally equivalent in $A(\tilde{S})$ to $\left(\mathbf{P}^{1} \times L\right) \cdot \tilde{X}$. We can then use the projection formula and adjunction to write the intersection in $A(\tilde{X})$ as an intersection in $A(\tilde{S})$. Thus,

$$
\mathcal{C}_{X} \cdot L=\left((d-n-2) H-K_{\tilde{S}}-\tilde{X}\right) \cdot\left(\mathbf{P}^{1} \times L\right) \cdot \tilde{X}
$$

Now to verify the theorem it suffices to directly evaluate the right hand side in the above equation using the intersection theory for $\tilde{S}$ developed earlier. Explicitly,

$$
\begin{aligned}
\mathcal{C}_{X} \cdot L= & \left((d-n-2) H-K_{\tilde{S}}-\tilde{X}\right) \cdot\left(\mathbf{P}^{1} \times L\right) \cdot \tilde{X} \\
= & \left((d-n-2) H-\left(-(n+1) H+\left(d_{S}-2\right) F\right)-(b H+F)\right) \\
& \quad \cdot\left(H^{n-1}-d_{S} H^{n-2} \cdot F\right) \cdot(b H+F) \\
= & \left(b\left(d_{S}-1\right) H+\left(1-d_{S}\right) F\right) \cdot\left(b H^{n}+\left(1-b d_{S}\right) H^{n-1} \cdot F\right) \\
= & b^{2}\left(d_{S}-1\right) H^{n+1}+b\left(d_{S}-1\right)\left(1-b d_{S}\right) H^{n} \cdot F+b\left(1-d_{S}\right) H^{n} \cdot F \\
= & 0 .
\end{aligned}
$$

Let $\pi$ be the sectional genus of $X$. A generic $(N-n+1)$-plane section of $X$ is a smooth curve, and $\pi$ is by definition the genus of this curve. $\pi$ can be computed by the formula

$$
2 \pi-2=K_{X} \cdot H^{n-1}+(n-1) H^{n} .
$$

A computation similar to that in the previous proposition gives

\section{Proposition 3.9.}

$$
\pi=\frac{1}{2} \frac{(d-1)(d-(N-n+1))}{(N-n)} .
$$

Corollary 3.10. Let $X^{n} \subset \mathbf{P}^{N}$ be a nonsingular, nondegenerate projective variety, and suppose that the generic $N-n+1$ plane section of $X$ is a nonsingular projectively normal curve. Then $H^{i}\left(X, \mathcal{O}_{X}(k)\right)=0$ for $1 \leq i \leq n-1$ and all $k \geq 0$. 
Let $X^{n} \subset \mathbf{P}^{N}$ be a nondegenerate variety of degree $d$ and $\phi: X^{\prime} \rightarrow X$ a resolution of $X$, i.e., $X^{\prime}$ is nonsingular and $\phi$ is a birational morphism. Such a resolution exists by Hironaka's resolution of singularities. Then the geometric genus of $X, p_{g}(X)=h^{n}\left(X^{\prime}, \mathcal{O}_{X^{\prime}}\right)$, is a well defined invariant of $X$. Let $M=\left[\frac{d-1}{N-n}\right]$ and let $\epsilon$ be defined by $d-1=M(N-n)+\epsilon$. Then there is a bound due to J. Harris on the geometric genus of $X$ in terms of $d, n$, and $N$ :

Theorem 3.11. [Ha1, page 44]

$$
p_{g}(X) \leq\left(\begin{array}{c}
M \\
n+1
\end{array}\right)(N-n)+\left(\begin{array}{c}
M \\
n
\end{array}\right) \epsilon,
$$

where the binomial coefficient $\left(\begin{array}{l}a \\ b\end{array}\right)=0$ when $b>a$.

This theorem motivates Harris' definition of Castelnuovo variety:

Definition 3.12. $X$ is a Castelnuovo variety if

$$
p_{g}(X)=\left(\begin{array}{c}
M \\
n+1
\end{array}\right)(N-n)+\left(\begin{array}{c}
M \\
n
\end{array}\right) \epsilon .
$$

(2) $d \geq n(N-n)+2$.

The second condition in the definition is intended to ensure that $p_{g}(X) \neq 0$. If $n=1$, Harris' bound is just the classical Castelnuovo bound for the genus of a space curve. The curves whose genus achieve this bound are Castelnuovo curves if $d \geq(N-n)+2$ and rational normal curves if $d=(N-n)+1$. In either case, these curves are projectively normal.

Now let $X^{n} \subset \mathbf{P}^{N}$ be a Roth variety of degree $d=b(N-n)+1$ contained in a rational normal scroll $S=S_{0,0, a_{1}, \ldots, a_{n-1}}$ with all $a_{i} \geq 1$ and singular locus $L$ such that $L \subset X \subset S$. It follows from Proposition 3.10 that if $b \geq 2$ then a generic $N-n+1$ plane section of $X$ is a Castelnuovo curve. If $b=1$ then $d=(N-n)+1$, and so by classification of varieties of minimal degree, $X$ is a rational normal scroll. (In fact, by Proposition 5.5, $N_{L / X}=\mathcal{O}_{L}\left(1-a_{1}\right) \oplus \cdots \oplus \mathcal{O}_{L}\left(1-a_{n-1}\right)$. It follows that $X$ is a rational normal scroll $S_{1, a_{1}, \ldots, a_{n-1}}$.) In either case, a standard argument shows that $X$ is projectively normal and $H^{i}\left(X, \mathcal{O}_{X}(k)\right)=0$ for $1 \leq i \leq n-1$ and $k \geq 0$.

Harris states that a nonsingular nondegenerate variety $X^{n} \subset \mathbf{P}^{N}$ is Castelnuovo if and only if its generic hyperplane section is. The "if" direction of this theorem is not quite true because condition (2) in the definition of Castelnuovo variety is not stable under taking hyperplane sections. For example, if $X \subset \mathbf{P}^{N}$ is a Roth surface of degree $d=2(N-n)+1$, then $X$ is not a Castelnuovo surface but a generic hyperplane section of $X$ is a Castelnuovo curve. However, provided that the degree of $X$ is in the required range, then Harris' result is correct. Namely,

Proposition 3.13. If $X^{n} \subset \mathbf{P}^{N}$ is a nonsingular nondegenerate variety of degree $d \geq n(N-n)+2$ and $Y$ is a generic hyperplane section of $X$, then $Y$ is Castelnuovo if and only if $X$ is.

To sum up, we've proved:

Theorem 3.14. Let $X^{n} \subset \mathbf{P}^{N}$ be a Roth variety of degree $d=b(N-n)+1$ and $S$ a rational normal scroll $S_{0,0, a_{1}, \ldots, a_{n-1}}$ with all $a_{i} \geq 1, \sum_{i=1}^{n-1} a_{i}=N-n$, and singular locus $L$ such that $L \subset X \subset S$. 
(1) If $b=1$ then $X$ is a rational normal scroll $S_{1, a_{1}, \ldots, a_{n-1}}$.

(2) If $b \geq 2$ then the generic $N-n+1$ plane section of $X$ is a Castelnuovo curve.

(3) $X$ is projectively normal.

(4) $H^{i}\left(X, \mathcal{O}_{X}(k)\right)=0$ for $1 \leq i \leq n-1$ and all $k \in \mathbf{Z}$.

(5) If $b \geq n+1$ then $X$ is a Castelnuovo variety.

Note that (3) and (4) imply that $X$ is arithmetically Cohen-Macaulay.

\section{Proof of the Main Reduction}

Proposition 4.1. Let $X^{n} \subset \mathbf{P}^{N}$ be a nonsingular, nondegenerate, linearly normal variety of dimension $n \geq 2$ and codimension $N-n \geq 2$. Let $p \neq q \in X$ be such that $S(p, X)=S(q, X)$ and let $L=\langle p, q\rangle$. Then,

(1) $L \subset X$.

(2) $S(L, X)$ is a rational normal scroll $S_{0,0, a_{1}, \ldots, a_{n-1}}$ with all $a_{i} \geq 1$ and $\sum_{i=1}^{n-1} a_{i}$ $=N-n$.

(3) $L$ is the singular locus of $S(L, X)$.

(4) $S(r, X)=S(L, X)$ for all $r \in L$.

In particular, $X$ is a Roth variety.

The main corollary is a classification of those varieties whose double-point divisor determines an ample linear system:

Theorem 4.2. Let $X^{n} \subset \mathbf{P}^{N}$ be a nonsingular, nondegenerate variety of codimension $N-n \geq 2$. Let $\left|\mathcal{C}_{X}\right|$ denote the linear system determined by the double-point divisor on $X$. Then the following are equivalent:

(1) $\left|\mathcal{C}_{X}\right|$ is ample.

(2) $\left|\mathcal{C}_{X}\right|$ separates points.

(3) $X$ is not an isomorphic projection of a Roth variety.

If, in addition, $X$ is linearly normal then (3) can be replaced by:

$\left(3^{\prime}\right) X$ is not a Roth variety.

Proof. By Remark 2.1 it suffices to prove the theorem when $X$ is linearly normal. Then $(3) \Longrightarrow(2)$ follows by Corollary 2.3 and Proposition $4.1,(2) \Longrightarrow(1)$ follows by Corollary 2.4, and (1) $\Longrightarrow$ (3) follows by Proposition 3.8 .

An alternative version of this theorem can be given which amounts to a classification of those varieties with certain highly degenerate associated secant varieties.

Theorem 4.3. Let $X^{n} \subset \mathbf{P}^{N}$ be a nonsingular, nondegenerate variety of codimension $N-n \geq 2$. Then the following are equivalent:

(1) There exists a positive dimensional subvariety $W$ of $X$ such that the secant variety $S(W, X)$ has dimension $n+1$.

(2) There is $p \neq q \in X$ such that $S(p, X)=S(q, X)$.

(3) $X$ is an isomorphic projection of a Roth variety.

If, in addition, $X$ is linearly normal then (3) can be replaced by:

$\left(3^{\prime}\right) X$ is a Roth variety.

Proof. Clearly it suffices to prove the theorem in the case when $X$ is linearly normal. (1) $\Longrightarrow$ (2) is clear. (2) $\Longrightarrow$ (3) is Proposition 4.1. (3) $\Longrightarrow$ (1) since if $X$ is a Roth variety with $L \subset X \subset S$, then $S(p, X)=S$ for all $p \in L$, so that $S(L, X)=S$ is $n+1$ dimensional. 
To begin the proof of Theorem 4.1, we quote a Bertini-like proposition that will be used often:

Proposition 4.4. [Ha2, Proposition 18.10] Let $X^{n} \subset \mathbf{P}^{N}$ be a nondegenerate variety of dimension $n \geq 1$ and let $H$ by a hyperplane intersecting $X$ generically transversally, i.e., for a generic point $p \in X \cap H, \tilde{T}_{p} X \not \subset H$. If $Y=X \cap H$, then $Y$ is nondegenerate as a (possibly reducible) subvariety of $H \cong \mathbf{P}^{N-1}$.

Proposition 4.5. Let $X^{n} \subset \mathbf{P}^{N}$ be a nonsingular, nondegenerate variety of dimension $n \geq 2$ and codimension $N-n \geq 2$. Let $Z^{n-1} \subset \mathbf{P}^{N-2} \subset \mathbf{P}^{N}$ be a variety and let $L$ be a line in $\mathbf{P}^{N}$ disjoint from $\mathbf{P}^{N-2}$. Let $\operatorname{Cone}_{L}(Z)$ be the cone over $Z$ with vertex $L$. Cone ${ }_{L}(Z)$ is an $n+1$ dimensional variety. Suppose that $X \subset$ Cone $_{L}(Z)$. Then $L \subset X$.

Proof. The proof is by induction on $n$. Suppose that $n=2$ and that $L \not \subset X$, so that $L \cap X=\left\{p_{1}, \ldots, p_{j}\right\}$ is a (possibly empty) finite set of points. Let $q_{i} \in$ $\tilde{T}_{p_{i}} X \backslash L$. Let $H$ be a generic hyperplane containing $L$ such that $q_{i} \notin H$ for all $i$. Let $Y=X \cap H$. By Bertini's theorem, $Y$ is nonsingular except possibly at $p_{1}, \ldots, p_{k}$. Since $\tilde{T}_{p_{i}} X \not \subset H$, it follows by the Jacobian criterion that $Y$ is nonsingular at $p_{i}$ for all $i$. Hence $Y$ is nonsingular. By Proposition 4.4, $Y \subset H$ is nondegenerate. By the Fulton-Hansen connectedness theorem [FL, Theorem 4.1], $Y$ is connected. Hence $Y$ is irreducible. On the other hand, if $H \cap Z=\left\{q_{1}, \ldots, q_{k}\right\}$, then, since $X \subset \operatorname{Cone}_{L}(Z), Y \subset H \cap \operatorname{Cone}_{L}(Z)=\left\langle L, q_{1}\right\rangle \cup \cdots \cup\left\langle L, q_{k}\right\rangle$. Since $Y$ is irreducible, $Y \subset\left\langle L, q_{i}\right\rangle$ for some $i$. This contradicts the nondegeneracy of $Y \subset H$.

If $n \geq 3$ and $L \not \subset X$, then by slicing with a generic hyperplane $H$ through $L$ we obtain $Y=H \cap X$. By the same argument as in the $n=2$ case it follows that $Y$ is nonsingular and a nondegenerate subvariety of $H$. By the induction hypothesis $L \subset Y$, which is a contradiction. Hence $L \subset X$.

Proposition 4.6. Let $X^{n} \subset \mathbf{P}^{N}$ be a nonsingular, nondegenerate variety of dimension $n \geq 2$ and codimension $N-n \geq 2$. Let $p \neq q \in X$ be such that $S(p, X)=S(q, X)$. Let $L=\langle p, q\rangle$. Then $L \subset X$ and $S(r, X)=S(L, X)$ for all $r \in L$.

Proof. Let $S=S(p, X)$ and let $\mathbf{P}^{N-2}$ be a generic $N-2$ plane in $\mathbf{P}^{N}$. Then $\mathbf{P}^{N-2} \cap L=\emptyset$, and $Z:=\mathbf{P}^{N-2} \cap S$ is an $n-1$ dimensional variety. Applying Proposition 2.6 to $W=\{p, q\}$, we see that $W \subset \tilde{T}_{p} X \cap \tilde{T}_{q} X$. Thus $L \subset \tilde{T}_{p} X \subset S$. Let $r \in Z$. We claim that $\langle L, r\rangle \subset S$. Since $r \in S$, either $r \in \tilde{T}_{p} X$ or the line $\langle p, r\rangle$ is a proper bisecant of $X$. In the first case, $\langle L, r\rangle \subset \tilde{T}_{p} X \subset S$. If $r \in \tilde{T}_{q} X$, then also $\langle L, r\rangle \subset S$. So suppose that $r \notin \tilde{T}_{q} X$ and that $\langle p, r\rangle$ is a proper bisecant of $X$. Then since $S(p, X)=S(q, X)$, for generic $w \in\langle p, r\rangle$, the line $\langle w, q\rangle$ is a proper bisecant line of $X$. Varying $w$, we obtain a curve $C \subset\langle L, r\rangle$ such that $C \neq L$. It follows that $\langle L, r\rangle \subset S$. Let $T$ be the cone over $Z$ with vertex $L$. Then $T \subset S$, and since $\operatorname{dim}(Z)=\operatorname{dim}(S)$ and $S$ is irreducible, it follows that $T=S$. Thus, by Proposition 4.5, $L \subset X$. Clearly $S(r, X)=S(L, X)$ for all $r \in L$.

Set up of notation 4.7. Now let $X^{n} \subset \mathbf{P}^{N}$ be a nonsingular, nondegenerate variety of dimension $n \geq 2$ and codimension $N-n \geq 2$. Let $L^{m} \subset X$ be a possibly reducible subvariety of $X$ such that $m \geq 1$ and $S(p, X)=S(L, X)$ for all $p \in L$. By Proposition 4.6 we can assume that $L$ is a linear space. Let $\mathbf{P}\left(V_{1}\right)$ be a generic $N-m-1$ plane in $\mathbf{P}^{N}$. Then $\mathbf{P}\left(V_{1}\right) \cap L=\emptyset$, and $Z:=\mathbf{P}\left(V_{1}\right) \cap S(L, X)$ is an 
$n-m$ dimensional variety. $Z \subset \mathbf{P}\left(V_{1}\right)$ is nondegenerate by Proposition 4.4 . Let Cone $_{L}(Z)$ be the cone over $Z$ with vertex $L$.

Proposition 4.8. With notation as in 4.7,

(1) If $q \in S(L, X)$, then $\langle L, q\rangle \subset S(L, X)$.

(2) $\operatorname{Cone}_{L}(Z)=S(L, X)$.

Proof. Let $q \in S(L, X)$. Then by Proposition 2.5, $S(L, X)=\bigcup_{p \in L} \tilde{T}_{p} X$, so there is $p \in L$ such that $q \in \tilde{T}_{p} X$. It follows that $\langle L, q\rangle \subset \tilde{T}_{p} X \subset S(L, X)$. Since $Z \subset S(L, X)$, it follows that $\mathrm{Cone}_{L}(Z) \subset S(L, X)$. On the other hand, $\operatorname{Cone}_{L}(Z)$ and $S(L, X)$ are both irreducible varieties of dimension $n+1$. Hence Cone $_{L}(Z)=$ $S(L, X)$.

Proposition 4.9. With notation as in 4.7, suppose that $n-m \geq 2$. Let $H$ be a generic hyperplane containing $L$. Then $Y=X \cap H$ is a nonsingular, nondegenerate variety, and $S(p, Y)=S(L, Y)$ for all $p \in L$.

Proof. Since $L \subset \bigcap_{p \in L} \tilde{T}_{p} X$ by Proposition 2.6 and since $L$ and $\mathbf{P}\left(V_{1}\right)$ are complementary linear spaces in $\mathbf{P}^{N}, \tilde{T}_{p} X \cap \mathbf{P}\left(V_{1}\right)$ is an $n-m-1$ plane for every $p \in L$. Moreover, since $S(L, X)=\bigcup_{p \in L} \tilde{T}_{p} X$, this plane is contained in $Z$. Let $H$ be a generic hyperplane containing $L$. By Bertini's theorem, $Y=X \cap H$ is a possibly reducible variety that is nonsingular off of $L$. By Proposition 4.4, $Y$ is nondegenerate. Moreover, by the Jacobian criterion, if $p \in L$ then $Y$ is nonsingular at $p$ if $\tilde{T}_{p} X \not \subset H$, which holds if $\tilde{T}_{p} X \cap \mathbf{P}\left(V_{1}\right) \not \subset H \cap \mathbf{P}\left(V_{1}\right)$. But $H \cap \mathbf{P}\left(V_{1}\right)$ is a generic hyperplane in $\mathbf{P}\left(V_{1}\right)$, which meets $Z$ in an $n-m-1 \geq 1$ dimensional variety that is not a linear space since $Z$ is not a linear space. Hence the $n-m-1$ plane $\tilde{T}_{p} X \cap \mathbf{P}\left(V_{1}\right)$ is not contained in $H \cap \mathbf{P}\left(V_{1}\right)$ for any $p \in L$. Thus $Y$ is nonsingular. Now by the Fulton-Hansen connectedness theorem, $Y$ is connected and thus $Y$ is irreducible. $S(p, Y)=S(L, Y)$ for all $p \in L$, since $S(p, X) \cap H=S(p, Y)$.

Let $X^{n} \subset \mathbf{P}^{N}$ be a nonsingular, nondegenerate projective variety. Let $L^{m}$ be a linear subspace contained in $X$ with $m \geq 1$, and let $\mathbf{P}\left(V_{1}\right)$ be an $n-m-1$ plane in $\mathbf{P}^{N}$ that does not meet $L$. Let $\pi_{L}: X--\rightarrow \mathbf{P}\left(V_{1}\right)$ be the rational map determined by projection from the center $L$ onto $\mathbf{P}\left(V_{1}\right)$. Let $\Gamma \subset X \times \mathbf{P}\left(V_{1}\right)$ be the graph of $\pi_{L} . \Gamma$ is also the blow up of $X$ along $L$. Let $E$ be the exceptional divisor of the blow up. We have the diagram

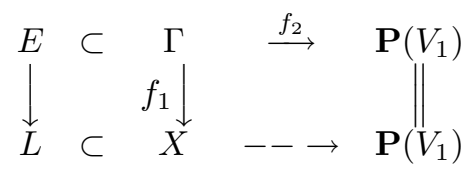

Proposition 4.10. With notation as in 4.7:

(1) $Z$ is an $n-m$ dimensional variety, not a linear space, and nondegenerate as a subvariety of $\mathbf{P}\left(V_{1}\right)$.

(2) $Z=\pi_{L}(X)$.

(3) $\left.f_{2}\right|_{E}: E \rightarrow Z$ is surjective.

Proof. (1) is part of the set up of 4.7. By Proposition $4.8(1), \pi_{L}(X) \subset Z$. Now, since $S(L, X)=\bigcup_{p \in L} \tilde{T}_{p} X$, 


$$
Z=S(L, X) \cap \mathbf{P}\left(V_{1}\right)=\bigcup_{p \in L}\left(\tilde{T}_{p} X \cap \mathbf{P}\left(V_{1}\right)\right)=\bigcup_{p \in L} f_{2}\left(f_{1}^{-1}(p)\right)=f_{2}(E) .
$$

Thus, (2) and (3) follow.

Proposition 4.11. With notation as in $4.7, L$ is a line.

Proof. Using Proposition 4.9, by repeatedly slicing by generic hyperplanes through $L$ we can assume that $n-m=1$. The blow up of $X$ along $L$ is then isomorphic to $X$, since $L$ is a divisor in $X$. Thus $L=E$, and we obtain a surjective morphism $L \cong E \stackrel{\left.f_{2}\right|_{E}}{\longrightarrow} Z$ from a projective space of dimension $m$ to the curve $Z$, and so $m=1$.

As mentioned in the introduction, the next proposition follows the main line of argument of the proof of [So, Lemma 1.1.1].

Proposition 4.12. Let $X \subset \mathbf{P}^{N}$ be a nonsingular, nondegenerate and linearly normal projective surface with $N \geq 3$. Let $L \subset X$ be a line such that $S(p, X)=$ $S(L, X)$ for all $p \in L$. Then $S(L, X)$ is a rational normal scroll $S_{0,0, N-2}$.

Proof. The blow up of $X$ along $L$ is isomorphic to $X$, since $L$ is a divisor in $X$. Thus, identifying $\Gamma$ with $X$ and $E$ with $L$, we obtain the diagram

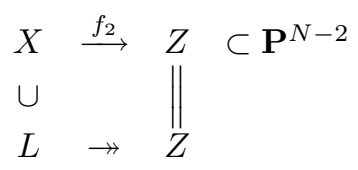

where $Z$ is a curve. Since $Z$ is nondegenerate, the morphism $f_{2}$ is the morphism determined by a base point free subsystem $\Lambda$ of the linear system $|H-L|$, and $\operatorname{dim}(\Lambda)=N-2$. Since $X$ is nondegenerate and linearly normal, $\operatorname{dim}|H|=N$. Since vanishing on the line $L$ imposes two conditions on linear forms, $\operatorname{dim}|H-L|=N-2$. Thus $\Lambda$ is the complete linear system $|H-L|$. Hence $Z$ is linearly normal. Since $\mathbf{P}^{1}=L \rightarrow Z$, we have that $Z \cong \mathbf{P}^{1}$ and that $Z$ is a nondegenerate rational normal curve of degree $N-2$. Since $S(L, X)=\operatorname{Cone}_{L}(Z)$, it follows that $S(L, X)$ is a rational normal scroll $S_{0,0, N-2}$.

Proposition 4.13. Let $X^{n} \subset P^{N}$ be a nonsingular, nondegenerate and linearly normal projective variety of codimension $N-n \geq 2$. Let $L \subset X$ be a line such that $S(p, X)=S(L, X)$ for all $p \in L$. Then $S(L, X)$ is a rational normal scroll $S_{0,0, a_{1}, \ldots, a_{n-1}}$ with all $a_{i} \geq 1$ and $N-n=\sum_{i=1}^{n-1} a_{i}$. Moreover, $L$ is the singular locus of the scroll.

Proof. The proof is by induction on $n$. The case of $n=2$ was done in the last proposition. So suppose that $n \geq 3$ and that the result is true for varieties of dimension less that $n$. Take a generic hyperplane $H$ containing $L$, and consider $Y=X \cap H$. By Proposition 4.9 we know that $Y$ is a nonsingular, nondegenerate variety with $S(p, Y)=S(L, Y)=S(L, X) \cap H$ for all $p \in L$. By the induction hypothesis, $Y$ is an isomorphic projection of a Roth variety. Thus, by Theorem $3.14(4), H^{1}\left(Y, \mathcal{O}_{Y}\right)=0$, and so $H^{1}\left(X, \mathcal{O}_{X}(-H)\right) \rightarrow H^{1}\left(X, \mathcal{O}_{X}\right)$ is surjective. But, by the Kodaira vanishing theorem, $H^{1}\left(X, \mathcal{O}_{X}(-H)\right)=0$, and so $H^{1}\left(X, \mathcal{O}_{X}\right)=0$. Hence $Y$ is linearly normal, and so $Y$ is a Roth variety and $S(L, Y)$ is a rational 
normal scroll. Since $S(L, Y)=S(L, X) \cap H$, the classification of varieties of minimal degree [Ha1, page 51] shows that $S(L, X)$ is a rational normal scroll.

Let $M$ be the singular locus of $S(L, X)$. It remains to verify that $M=L$. By Proposition $4.8(2)$ it follows that $L \subset M$. Since $S(L, X)$ is a rational normal scroll, $S(L, X)$ is a cone with vertex $M$. Pick a line $L^{\prime}$ in $M$. Then $S(L, X)$ is also a cone over a rational normal scroll $Z^{n-1}$ with vertex $L^{\prime}$ such that $\langle Z\rangle$ and $L^{\prime}$ are complementary linear subspaces of $\mathbf{P}^{N}$. Hence by Proposition 4.5, $L^{\prime} \subset X$. Hence $M \subset X$. Clearly $S(p, X)=S(M, X)$ for all $p \in M$. Hence $M=L$ by Proposition 4.11 .

\section{Applications of the Classification AND FURTHER PROPERTIES OF ROTH VARIETIES}

Theorem 5.1. Let $X^{n} \subset \mathbf{P}^{N}$ be a Roth variety of degree $d=b(N-n)+1$ and $S$ a rational normal scroll $S_{0,0 . a_{1}, \ldots, a_{n-1}}$ with all $a_{i} \geq 1, \sum_{i=1}^{n-1} a_{i}=N-n$, and singular locus $L$ such that $L \subset X \subset S$. Then,

(1) If $b \geq 2$ then the intersection of all quadrics containing $X$ is $S$. Hence the associated scroll $S$ is uniquely determined by $X$.

(2) The generic $N-n+1$ plane section of $X$ containing $L$ is

$$
L \cup C_{1} \cup \cdots \cup C_{N-n},
$$

where $C_{i}$ are nonsingular plane curves of degree $b, C_{i} \neq L$, and if $p_{i} \in C_{i} \backslash L$ then $C_{i} \subset\left\langle L, p_{i}\right\rangle$. Moreover, $C_{i} \cap C_{j}=\emptyset$ if $i \neq j$, and $C_{i} \sim C_{j}$ in $A(X)$.

(3) If $n \geq 3$, the generic hyperplane section of $X$ containing $L$ is a Roth variety.

Proof. We will prove (2) first. Using Proposition 4.9, by taking successive hyperplane sections through $L$ we can assume that $n=2$. Then projection from $L$ extends to a morphism $\pi_{L}: X \rightarrow S_{N-2} \subset \mathbf{P}^{N-2}$ as in the proof of Proposition 4.12. Let $H^{\prime}$ be a generic hyperplane in $\mathbf{P}^{N-2}$ and let $H=\left\langle L, H^{\prime}\right\rangle$. Suppose that $H^{\prime} \cap S_{N-2}=\left\{q_{1}, \ldots, q_{N-2}\right\}$. Let $C_{i}=\pi_{L}^{-1}\left(q_{i}\right)$. Then since $S_{N-2} \cong \mathbf{P}^{1}, C_{i} \sim C_{j}$ for all $i, j$. Also, $C_{i} \cap C_{j}=\emptyset$ if $i \neq j$. Since $\pi_{L}$ is projection from $L, C_{i} \subset\left\langle L, q_{i}\right\rangle$. Also, $C_{i}$ is nonsingular by Bertini's theorem. Thus $H \cap X=L \cup C_{1} \cup \cdots \cup C_{N-2}$. Thus, $\operatorname{deg}\left(C_{i}\right)=b$ for all $i$. This proves (2). Now by (2) it is clear that if $b \geq 1$ any quadric that contains $X$ also contains $S$. Since the ideal of $S$ is generated by quadrics [ACGH, page 96], (1) follows. (3) follows as in the proof of Proposition 4.13 .

The next proposition follows by a computation similar to that of Proposition 3.8 .

Proposition 5.2. If $X^{n} \subset \mathbf{P}^{N}$ is a Roth variety of degree $d=b(N-n)+1$, then

$$
\mathcal{C}_{X}^{n}=(d-b-1)^{n}(d-n) .
$$

Corollary 5.3. If $X^{n} \subset \mathbf{P}^{N}$ is a nonsingular nondegenerate variety of codimension $N-n \geq 2$, then $\mathcal{C}_{X}$ is big unless $X$ is the rational normal scroll $S_{1, \ldots, 1}$ (n 1's with $n \geq 3$ ). (This is just $\mathbf{P}^{1} \times \mathbf{P}^{n-1}$ embedded into $\mathbf{P}^{2 n-1}$ by the Segre embedding.)

Proof. If $\mathcal{C}_{X}$ is ample then $\mathcal{C}_{X}$ is big, so we can assume that $\mathcal{C}_{X}$ is not ample. We can also first assume that $X$ is linearly normal, since if $X^{\prime}$ is an isomorphic projection of $X$ then $\mathcal{C}_{X}^{n}=\mathcal{C}_{X^{\prime}}^{n}$. Thus $X$ is a Roth variety. So by the above proposition, $\mathcal{C}_{X}^{n} \leq 0 \Longleftrightarrow d \leq n$. But $d=b(N-n)+1$, and since $N-n=\sum_{i=1}^{n-1} a_{i} \geq n-1$ 
we get that $b(n-1)+1 \leq n$, and so $b=1$. Then $d \leq n$ implies $(N-n)+1 \leq n$, so $\sum_{i=1}^{n-1} a_{i} \leq n-1$ and thus $a_{i}=1$ for all $i$. Thus $X$ is a Roth variety with $b=1$ contained in $S_{0,0,1, \ldots, 1}$, and so the result follows when $X$ is linearly normal. Since the secant variety of $\mathbf{P}^{1} \times \mathbf{P}^{n-1} \subset \mathbf{P}^{2 n-1}$ is $\mathbf{P}^{2 n-1}$, it follows that $X$ must be linearly normal.

Corollary 5.4. If $X^{n} \subset \mathbf{P}^{N}$ is a nonsingular, nondegenerate variety of degree $d$ and codimension $N-n \geq 2$, then

$$
H^{i}\left(X, \mathcal{O}_{X}((d-n-2) H)\right)=0 \quad \text { for all } i \geq 1 .
$$

Proof. $C_{X}$ is nef, and if $C_{X}$ is also big then since $K_{X}+\mathcal{C}_{X}=(d-n-2) H$ the result follows by the Kawamata-Viehweg vanishing theorem [V]. If $C_{X}$ is not big, then a simple direct calculation with the exceptional cases given in the previous corollary completes the proof.

We can also compute the normal bundle of the line in a Roth variety (see my thesis for the proof [I, Proposition 5.9]).

Proposition 5.5. Let $X^{n} \subset \mathbf{P}^{N}$ be a Roth variety of degree $d=b(N-n)+1$ and $S$ a rational normal scroll $S_{0,0 . a_{1}, \ldots, a_{n-1}}$ with all $a_{i} \geq 1, \sum_{i=1}^{n-1} a_{i}=N-n$, and singular locus $L$ such that $L \subset X \subset S$. Then

$$
N_{L / X}=\mathcal{O}_{L}\left(1-b a_{1}\right) \oplus \cdots \oplus \mathcal{O}_{L}\left(1-b a_{n-1}\right) .
$$

In particular, $c_{1}\left(N_{L / X}\right)=n-d$.

Let $S^{k}(X) \subset \mathbf{P}^{N}$ be the $k^{t h}$ secant variety of $X$, i.e., the union of the secant $k$-planes of $X$. The maximum dimension of $S^{k}(X)$ is $\min (n(k+1)+k, N)$. Then $S^{k}(X)$ is degenerate if $n(k+1)+k-\operatorname{dim} S^{k}(X)>0$.

Proposition 5.6. If $X^{n} \subset \mathbf{P}^{N}$ is a Roth variety, then $S^{k}(X)$ is degenerate for $k \geq 2$.

Proof. $L \subset X \subset S_{0,0, a_{1}, \ldots, a_{n-1}}$, where $\sum a_{i}=N-n$. Thus,

$$
S^{k}(X) \subset S^{k}\left(\operatorname{Join}\left(L, S_{a_{1}, \ldots, a_{n-1}}\right)\right) \subset \operatorname{Join}\left(L, S^{k}\left(S_{a_{1}, \ldots, a_{n-1}}\right)\right) .
$$

The right hand side has dimension $\leq 2+(n-1)(k+1)+k=n(k+1)+1$, which is less than $n(k+1)+k$ if $k \geq 2$.

Finally, we have some concluding remarks.

Remark 5.7. Analogous to the study of Roth varieties, we can consider $X^{n} \subset \mathbf{P}^{N}$ a nonsingular, nondegenerate variety such that there is an $n+1$ dimensional rational normal scroll $S=S_{0, a_{1}, \ldots, a_{n}}$ with all $a_{i} \geq 1, \sum a_{i}=N-n$ and singular point $p$ such that $p \subset X \subset S$. Mutatis mutandis, all the results of Section 3 for Roth varieties (except those expressing properties of the line $L$ in the Roth variety) hold for the above varieties and can be proved using the techniques of that section. In particular, Theorem 3.7 and Theorem 3.14 hold.

Remark 5.8. Let $X^{n} \subset \mathbf{P}^{N}$ be a nonsingular, nondegenerate variety of codimension $N-n \geq 2$. The most obvious question left unresolved in this work is to understand when the linear system $\left|\mathcal{C}_{X}\right|$ determined by the double-point divisor on $X$ is very ample. If $X$ is not a Roth variety and $\left|\mathcal{C}_{X}\right|$ is not very ample, then $\left|\mathcal{C}_{X}\right|$ separates points but does not separate tangent directions. We don't know if such examples exist. An even more interesting question is to understand when tangent directions 
can be separated using only geometrically constructed sections of $\left|\mathcal{C}_{X}\right|$, i.e., sections of the form $\mathcal{C}_{X, \Lambda}$ for some center of projection $\Lambda$.

\section{REFERENCES}

[ACGH] E. Arbarello, M. Cornalba, P.A. Griffiths, and J.Harris, Geometry of algebraic curves, vol. 1, Springer-Verlag, New York, 1984. MR 86h:14019

[BM] D. Bayer and D. Mumford, What can be computed in algebraic geometry?, Computational algebraic geometry and commutative algebra (Cortona, 1991), Sympos. Math., XXXIV, Cambridge Univ. Press, 1993, pp. 1-48. MR 95d:13032

[Ein] L.Ein, The ramification divisor for branched coverings of $\mathbf{P}^{n}$, Math. Ann. 261 (1982), 483-485. MR 84c: 14009

[EH] D. Eisenbud and J. Harris, On varieties of minimal degree (a centennial account), Proceedings of the Symposia in Pure Math, vol 46, part 1, A.M.S, 1987, pp. 3-13. MR 89f: 14042

[FL] W. Fulton and R. Lazarsfeld, Connectivity and its applications in algebraic geometry, Algebraic geometry (Chicago, IL, 1980), Lecture Notes in Math., vol. 862, SpringerVerlag, New York, 1981, pp. 26-92. MR 83i:14002

[Ha1] J. Harris, A bound on the geometric genus of projective varieties, Ann. Scuola Norm. Sup. Pisa Cl. Sci., Ser. 48 (1981), 35-68. MR 82h:14010

[Ha2] Joe Harris, Algebraic Geometry - a First Course, Graduate Texts in Math. Vol. 133, Springer-Verlag, New York, 1992. MR 93j:14001

[Hart] Robin Hartshorne, Algebraic Geometry, Graduate Texts in Math. Vol. 52, SpringerVerlag, New York, 1977. MR 57:3116

[HOV] K. Hulek, C. Okonek, and A. Van de Ven, Multiplicity-2 structures on Castelnuovo surfaces, Ann. Scuola Norm. Sup. Pisa Cl. Sci., Ser. 413 (1986), 427-448. MR 88c: 14052

[I] Bo Ilic, Geometric properties of the double-point divisor, Thesis, Columbia University, 1995, E-prints: alg-geom/950309.

[Io] Paltin Ionescu, Embedded projective varieties of small invariants, Algebraic Geometry, Bucharest 1982, Lecture Notes in Math., vol. 1056, Springer-Verlag, 1984, pp. 142-186. MR 85m:14024

[Kl] Steven L. Kleiman, The enumerative theory of singularities, Real and Complex Singularities, Oslo 1976, P. Holm (ed.), Sijthoff and Noordhoff, Alphen aan den Rijn, 1977, pp. 297-396. MR 58:27960

[Lar] M. E. Larsen, On the topology of complex projective manifolds, Invent. Math. 19 (1973), 251-260. MR 47:7058

[Mum] David Mumford, The Red Book of Varieties and Schemes, Lecture Notes in Math., vol. 1358, Springer-Verlag, Berlin, 1988. MR 89k:14001

[Ro] Leonard Roth, On the projective classification of surfaces, Proc. London Math. Soc. (2) 42 (1937), 142-170.

[So] Andrew John Sommese, Hyperplane sections of projective surfaces. I: The adjunction mapping, Duke Math. Journal 46 (1979), 377-401. MR 82f:14033

[V] E. Viehweg, Vanishing theorems, Jour. Reine Angew. Math. 335 (1982), 1-8. MR 83m:14011

[Zak] F. L. Zak, Tangents and secants of algebraic varieties, Translations of Math. Monographs vol. 127, American Math. Society, 1993. MR 94i:14053

Department of Mathematics, Columbia University, New York, New York 10027

Current address: Department of Mathematics, University of California, Los Angeles, California 90024

E-mail address: ilic@math.ucla.edu 